\title{
I farmaci per lo scompenso cardiaco: criteri di scelta razionale e costo-efficace
}

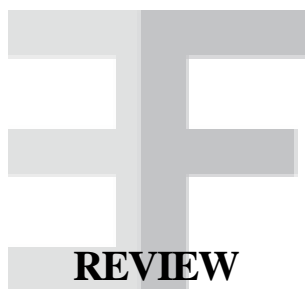

Francesco Vittorio Costa*, Lorenzo Pradelli§

\begin{abstract}
Chronic heart failure (CHF) is the final phase of many common cardiovascular diseases. Consequently, it represents a frequent clinical condition: it's estimated that in developed countries, Italy included, its prevalence exceeds $3 \%$. CHF is also burdensome from an economical point of view, as it absorbs more than $2 \%$ of the Italian total health care budget. The main cost driver in CHF, accounting for approximately two thirds of its total expense, is represented by hospital admissions for relapse. The most frequent reason for relapse, in turn, is inadequate treatment, intended both as low patient compliance to prescribed drug regimens and as inappropriate prescribing.

Evidence-based guidelines for the optimal pharmacological treatment of CHF have been developed and are constantly updated, and it's demonstrated that the stricter the adherence to these recommendations, the better the clinical and economic outcomes. Pharmacoeconomic studies conducted on the use of ACE-inhibitors and beta-blockers, in particular, have shown that correct therapeutic strategies can be cost-saving in CHF management, besides providing important clinical benefits.

The expansion of generic drug market has brought by a reduction in pharmaceutical prices, allowing to offer the benefits of these highly effective, and cost-effective, treatments for CHF to a larger number of patients, without increasing the global pharmaceutical expense, but probably reducing the total economical burden of the disease.
\end{abstract}

Keywords: chronic heart failure (CHF), ACE-inhibitors, beta-blockers, generic drugs Farmeconomia e percorsi terapeutici 2005; 6 (3): 215-226

\section{INTRODUZIONE}

Lo scompenso cardiaco costituisce l'ultimo stadio di gran parte delle malattie cardiovascolari, ed è quindi un evento clinico di riscontro assai comune. La sua incidenza è costantemente aumentata negli ultimi decenni fino a rappresentare oggi un vero e proprio problema di salute pubblica che assorbe un enorme e crescente impegno di risorse sanitarie [1- 3]. Paradossalmente, l'incidenza dello scompenso cardiaco sembra aumentare con la riduzione della mortalità per infarto e per altre malattie cardiovascolari. Ciò è spiegabile con l'aumentato numero di soggetti che sopravvivono all'infarto con danni miocardici residui di una qualche entità e che, col tempo, sviluppano un'insufficienza cardiaca.

\section{EPIDEMIOLOGIA}

L'incidenza dello scompenso sintomatico è attualmente pari allo $0,5 \%-1 \%$ e la prevalenza pari all' $1-2 \%$ della popolazione totale. Prevalenza ed incidenza aumentano in modo quasi esponenziale con l'età, per questo motivo l'età media d'insorgenza dei sintomi si colloca intorno ai 76 anni. La prevalenza dello scompenso cardiaco è pari all' $1 \%$ nelle persone di età compresa tra 50-59 anni, al 9\% tra 8089 anni.

Nel 1997 l'età media dei pazienti ricoverati in Lombardia con una diagnosi di scompenso cardiaco era di 71,8 anni per i maschi e di 78,3 per le femmine e il $70 \%$ dei ricoverati era costituito da ultrasettantenni. Inoltre, mentre il numero dei ricoveri in ospedale per scompenso cardiaco nei pazienti di età inferiore ai 65 anni è rimasto stabile, esso è raddoppiato nei più anziani.

Attualmente, lo scompenso cardiaco è la prima diagnosi di dimissione per malattia cardiovascolare, rappresenta la causa più frequente di ricovero nell' anziano ed è la patologia cardiovascolare più dispendiosa sul piano economico nei paesi occidentali. La ragione della frequenza dei ricoveri è riconducibile al fatto che lo scompenso cardiaco è una malattia di tipo cronico, caratterizzata dal succedersi di periodi di stabilità e instabilità clinica, con frequenti recidive.
*Professore associato in Medicina Interna Università degli Studi di Bologna

\$Centro di ricerche farmacoeconomiche Advanced Research Srl 


\section{COSTI DELLO SCOMPENSO CARDIACO}

Dall'analisi delle schede di dimissione ospedaliera risulta che in Italia nel 1999 tra le malattie dell'apparato cardiovascolare, classificate come MDC 5 (1.331.396), il DRG 127 (insufficienza cardiaca e shock) risulta al primo posto per frequenza (175.420) e per numero di giornate di degenza (con una degenza media di 10,3 giorni) [4].

Il rischio di riospedalizzazione nei 6 mesi successivi ad un primo ricovero è elevato (36$44 \%$ secondo le casistiche) ed è particolarmente frequente nei pazienti più anziani. A differenza di altre cardiopatie di comune rilievo, la mortalità per scompenso cardiaco, corretta per l'età, è in aumento.

La prognosi dello scompenso cardiaco risulta infatti molto sfavorevole quando la causa sottostante non sia correggibile. In circa la metà dei pazienti in cui sia stata posta diagnosi di scompenso cardiaco, il decesso avviene entro 4 anni, mentre in metà di quelli affetti da scompenso cardiaco grave il decesso avviene entro 1 anno.

\section{LA FORMAZIONE DEL COSTO DI MALATTIA}

In tutti i paesi occidentali i costi relativi ai ricoveri ospedalieri costituiscono la spesa più rilevante nella gestione della malattia [5-7]. In Italia oltre 127.000 pazienti vengono ricoverati ogni anno in ospedale con diagnosi di scompenso cardiaco. L'insufficienza cardiaca rappresenta il primo DRG, per numerosità, nell' ambito delle malattie trattate in Medicina Interna, costituendo 1'8\% di tutti i ricoveri.

I costi stimati per l'assistenza ai pazienti con scompenso superano il $2 \%$ della spesa

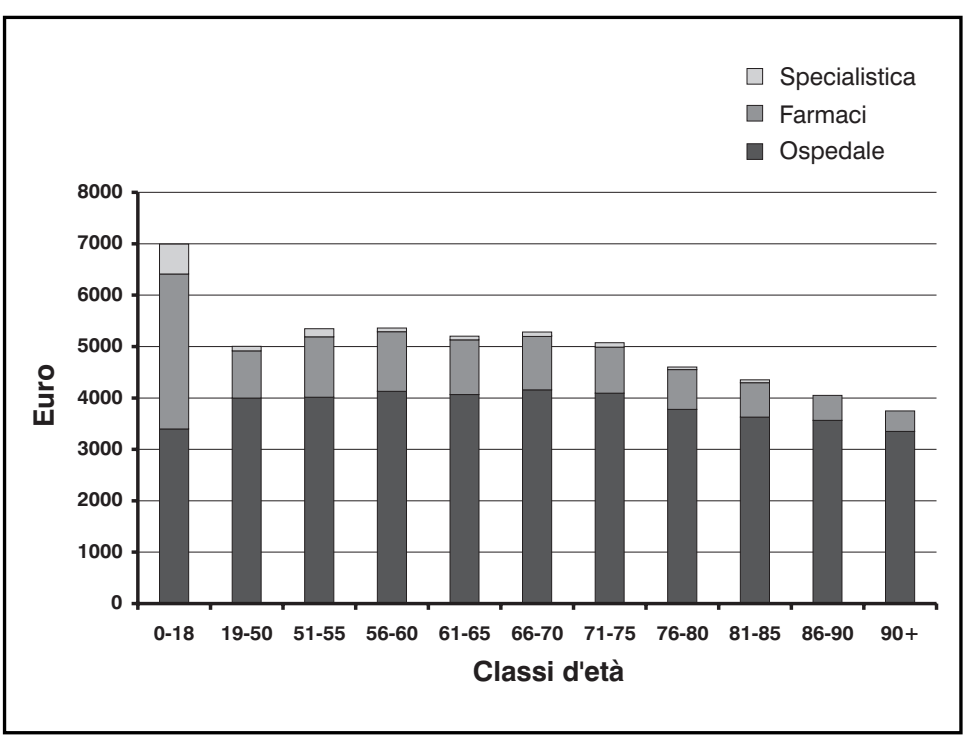

Figura 1

Costo medio per paziente con scompenso cardiaco rispetto alla classe d'età [8] sanitaria globale e circa i due terzi di questa sono rappresentati proprio dai costi derivanti dai ricoveri. Uno studio condotto nelle Marche [8] ha evidenziato come lo scompenso assorba il 2,5-2,6\% di tutte le risorse sanitarie, con un costo medio per paziente intorno ai 5.000 euro, di cui la spesa per il ricovero ospedaliero costituisce circa l' $80 \%$ del totale (Figura 1).

Esperienze analoghe sono state rilevate in molti altri paesi in cui il costo del ricovero ospedaliero costituisce dal 60 al $70 \%$ dei costi complessivi riferibili allo scompenso (Tabella I) [9].

\section{STRATEGIE TERAPEUTICHE RAZIONALI: IL RUOLO DELLE LINEE GUIDA}

Appare dunque logico che ogni tentativo di controllo della spesa debba passare attraverso la riduzione dell'incidenza delle riacutizzazioni, e quindi delle ospedalizzazioni. Ciò ottiene il duplice effetto di migliorare la qualità di vita dei pazienti e di far risparmiare risorse economiche, ma è ottenibile solo ottimizzando la terapia individuale e raggiungendo elevati livelli di compliance al trattamento. La causa più comune di riacutizzazione è, infatti, la cattiva aderenza al trattamento. Uno studio eseguito negli ospedali di Pordenone ha evidenziato come circa il $40 \%$ dei pazienti ricoverati per recidiva di scompenso dichiarasse una scarsa aderenza al regime terapeutico e il $25 \%$ circa scarsa compliance ai farmaci [9] (Tabella II). Essendo noto che i pazienti tendono a sovrastimare la loro compliance, appare evidente che i dati reali sono probabilmente ancora peggiori e che l'aderenza allo schema terapeutico costituisce il vero problema nella gestione di questi soggetti.

La terapia farmacologica dello scompenso cardiaco è rivolta a migliorare la sopravvivenza, i sintomi, la qualità di vita, i parametri emodinamici e la funzione ventricolare. Attualmente questi obiettivi sono perseguibili tramite l'azione combinata di vari farmaci capaci di ridurre il grado di attivazione neurormonale e di normalizzare le alterazioni del carico emodinamico. Le modalità per un corretto impiego di diuretici, ACE-inibitori, beta-bloccanti, digitalici, spironolattone, nitroderivati, sono chiaramente e dettagliatamente esposte nelle linee guida prodotte dalle società scientifiche.

È riconosciuto che tali linee guida abbiano dei limiti, dovuti soprattutto al fatto che esse si basano prevalentemente su studi condotti presso strutture cardiologiche ospedaliere, alle quali di solito afferiscono i pazienti più giovani, più complessi o più gravi. In altre parole, un sottogruppo selezionato di malati con scompenso cardiaco che è diverso dalla grande massa di pazienti scompensati. Va ricorda- 
to, infatti che nel nostro Paese (come negli altri Stati occidentali) circa l' $80 \%$ dei pazienti con scompenso cardiaco non viene ricoverato in strutture cardiologiche, bensì di Medicina Generale o di Geriatria.

Ciononostante, le linee guida per il trattamento dello scompenso cardiaco costituiscono, vista la complessità della materia, uno strumento indispensabile per impostare una corretta terapia farmacologica $[10,11]$. Questi documenti, aggiornati periodicamente, indicano $i$ comportamenti da tenere, in linea con i risultati dei trial clinici randomizzati, e riportano i pareri degli esperti su argomenti ancora controversi.

Una descrizione completa delle linee guida per la terapia dello scompenso esula dagli obiettivi di questa rassegna, ma è comunque opportuno evidenziare alcuni aspetti della terapia che dovrebbero e potrebbero essere migliorati. La lettura dei dati forniti dall'Italian Network on Congestive Heart Failure IN-CHF database dell'ANMCO [12] consente oggi di formulare alcune considerazioni in merito all'approccio dei cardiologi nei riguardi di questa patologia. I pattern prescrittivi possono essere considerati adeguati rispetto alle linee guida, ma solo dal punto di vista qualitativo.

\begin{tabular}{lccc}
\hline \multicolumn{4}{c}{ Epidemiologia dei costi } \\
Nazione & Costo & $\begin{array}{c}\text { \% dovuta alla } \\
\text { ospedalizzazione }\end{array}$ & $\begin{array}{c}\text { \% della spesa } \\
\text { sanitaria globale }\end{array}$ \\
\hline Regno Unito (1991) & $£ 360$ milioni & 60 & 1,2 \\
Olanda (1988) & Fl 460 milioni & 70 & 1,0 \\
Francia (1991) & FF 11,4 miliardi & 64 & 1,9 \\
USA (1989) & \$ 9 miliardi & 71 & 1,5 \\
\hline
\end{tabular}

Tabella I

Costi dello scompenso cardiaco in vari paesi e \% riferibile ai costi del ricovero

\begin{tabular}{lc}
\multicolumn{1}{c}{ Cause } & $\%$ \\
\hline Non aderenza al regime terapeutico & 41,9 \\
Scarsa compliance verso i farmaci & 23,5 \\
Ischemia miocardica & 13,4 \\
Aritmie & 6,1 \\
Ipertensione arteriosa non controllata & 5,6 \\
Terapia inadeguata & 12,3 \\
\hline
\end{tabular}

\section{Tabella II}

Cause di riospedalizzazione in pazienti con scompenso cardiaco [9]

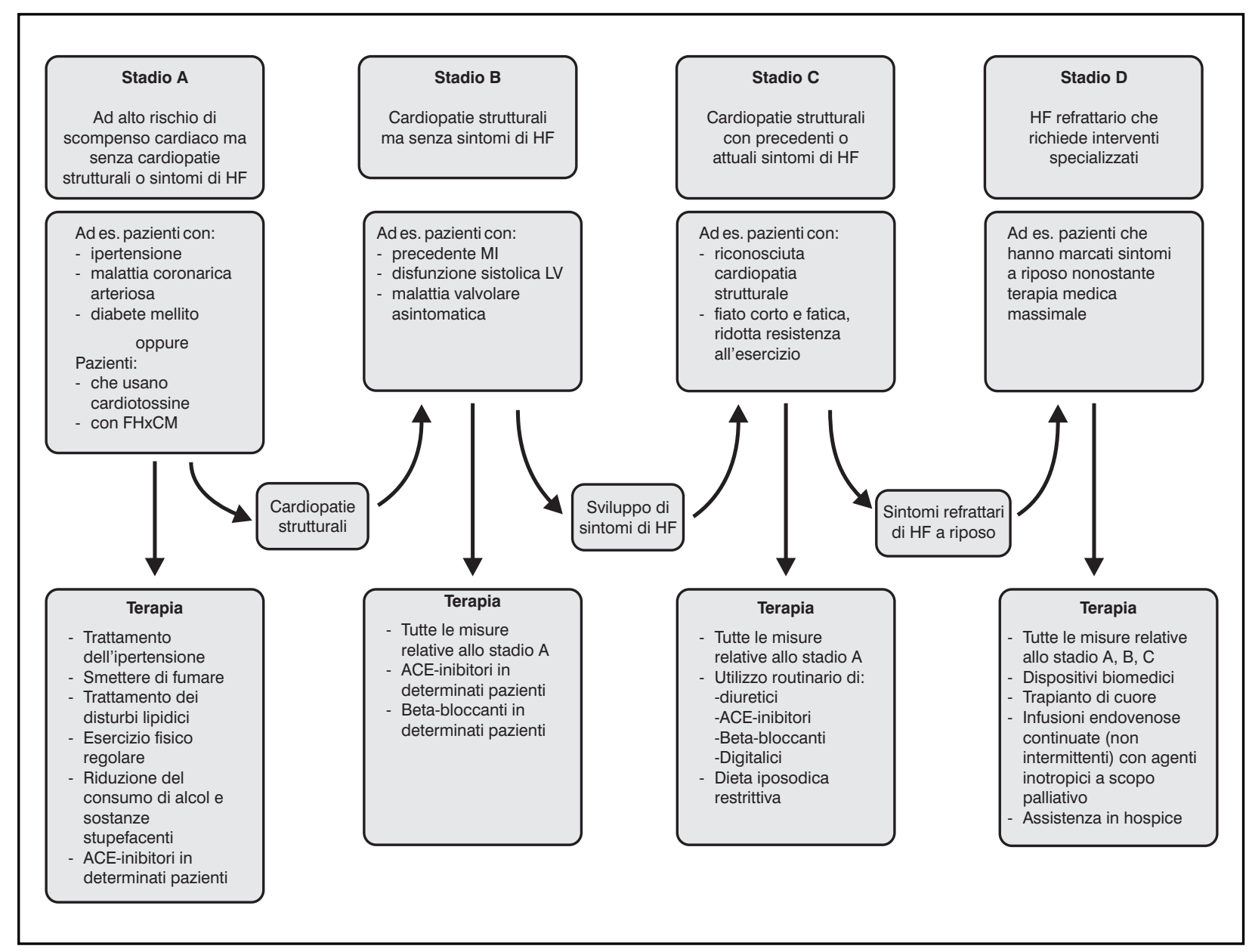

Figura 2

Stadi nell'evoluzione dello scompenso cardiaco e terapie raccomandate per singolo stadio

$\mathrm{HF}=$ scompenso cardiaco; $\mathrm{FHxCM}=$ storia familiare di cardiopatia; $\mathrm{Ml}=$ infarto del miocardio; $\mathrm{LV}=$ ventricolo sinistro 
Risulta infatti che le posologie dei trattamenti raccomandati sono inferiori a quelle utilizzate nei trial che ne hanno dimostrato l'efficacia e che una parte dei pazienti in cui ci sarebbe l'indicazione all'impiego di certi farmaci, in realtà non li riceve. Un qualche segno di miglioramento è tuttavia rilevabile, in quanto prescrizioni e relative posologie si stanno modificando nel tempo con un profilo che sembra via via più aderente alle linee guida.

Uno studio recente ha valutato le conseguenze dell'aderenza alle linee guida da parte dei cardiologi sullo scompenso e sul tasso di ricoveri. L'aderenza è stata valutata per le diverse classi di farmaci utilizzati e si è osservato che risultava migliore per gli ACE-I ( $88 \%)$ o per i diuretici (82\%) piuttosto che per i beta-bloccanti $(58 \%)$ i digitalici (52\%) e lo spironolattone $(36 \%)$. Si è anche osservato che, mano a mano che la compliance alle linee guida si riduce, il tasso di ricoveri cresce, passando dal $6,7 \%$ nei casi in cui le linee guida vengono seguite in maniera ottimale al $20,6 \%$ nei soggetti in cui non vengono applicate. Si dimostra così che l'aderenza alle linee guida da parete dei medici è un forte predittore delle ospedalizzazioni [13].

Questi risultati sono in linea con quelli ottenuti in un altro studio randomizzato condotto nel Veneto [14], che ha valutato, in pazienti dimessi dall'ospedale per un episodio acuto di scompenso, gli effetti di un trattamento basato sulle linee guida applicate in maniera intensiva e associato ad un programma di dieta e di informazione di pazienti e familiari. Si è osservato che nel gruppo trattato intensivamente rispetto ai soggetti seguiti in maniera standard, le recidive si dimezzano e i costi totali si riducono di circa il $35 \%$ (Tabella III).

$\mathrm{Nel}$ gruppo trattato intensivamente la spesa farmaceutica è stata circa il doppio di quella dei soggetti di controllo, e ciò a causa dell'impiego in un numero maggiore di pazienti e a dosaggi più alti, di beta-bloccanti, ACE-inibitori, inibitori dell' angiotensina e spironolattone. Ciò nonostante, i costi globali si sono sensibilmente ridotti nei soggetti trattati in maniera più aggressiva. Questo studio conferma che il trattamento eseguito a dosi corrette e con i farmaci adeguati, consente, anche nel breve periodo, risparmi sensibili qualora si considerino tutte le voci di costo della patologia e non, come spesso viene fatto dagli amministratori, la sola spesa farmaceutica, su cui spesso, sbagliando bersaglio, ci si accanisce. Appare quindi evidente che il metodo migliore per migliorare la salute e la qualità di vita dei pazienti e al contempo di risparmiare risorse finanziarie, è quello di trattarli in maniera adeguata e di facilitarne l'accesso a strutture ambulatoriali o di day-hospital in cui sia possibile prevenire i ricoveri mediante un controllo periodico e costante.

\section{PRINCIPALI CLASSI DI FARMACI DISPONIBILI: EFFICACIA E COSTO- EFFICACIA}

Esiste quindi, non un problema di eccessiva spesa farmaceutica, ma semmai un problema di sotto utilizzazione dei farmaci intesa sia come mancata prescrizione a pazienti che potrebbero giovarsene, sia come utilizzazione di dosi inferiori a quelle di provata efficacia.

\begin{tabular}{|c|c|c|c|c|c|c|}
\hline & \multicolumn{3}{|c|}{ Gruppo di studio } & \multicolumn{2}{|c|}{ Gruppo di controllo } & \multirow[t]{2}{*}{ Differenza } \\
\hline & $\begin{array}{l}\text { Costo } \\
\text { unitario }\end{array}$ & N. & $\begin{array}{l}\text { Costo } \\
\text { totale }\end{array}$ & N. & $\begin{array}{l}\text { Costo } \\
\text { totale }\end{array}$ & \\
\hline Costi ospedalieri & & & 32.294 & & 81.592 & -49.298 \\
\hline Riospedalizzazioni per DRG 127 & & 16 & & 31 & & \\
\hline Giornate di degenza & 219,00 & 136 & 29.784 & 365 & 79.935 & -50.151 \\
\hline Day-hospital (1 accesso di 3 ore) & 156,90 & 16 & 2.510 & 0 & 0 & 2.510 \\
\hline Accessi al Pronto Soccorso (senza ricovero) & 276,10 & 0 & 0 & 6 & 1.657 & -1.657 \\
\hline Costi extraospedalieri & & & 37.148 & & 12.608 & 24.540 \\
\hline Terapia farmacologica & & & 19.099 & & 9.872 & 9.227 \\
\hline Visite cardiologiche & 18,60 & 620 & 11.532 & 36 & 670 & 10.862 \\
\hline Ecocardiogrammi & 103,30 & 39 & 4.029 & 20 & 2.066 & 1.963 \\
\hline Tempo infermieristico (ore) & 12,50 & 199 & 2.488 & 0 & 0 & 2.488 \\
\hline Totale & & & 69.442 & & 94.200 & -24.758 \\
\hline
\end{tabular}

Tabella III

Spesa sanitaria (euro) nel trimestre post-dimissione nel gruppo di studio e nel gruppo di controllo [14] 


\begin{tabular}{lcccc}
\hline & Placebo $(\mathbf{n}=\mathbf{3 9 8})$ & Carvedilolo $(\mathbf{n}=\mathbf{6 9 6})$ & Differenza \% & P \\
\hline Per paziente & & & & \\
Giorni in ospedale & $3,08 \pm 11,72$ & $1,56 \pm 5,70$ & $-49 \%$ & .019 \\
Giorni in ICU/CCU & $1,46 \pm 9,69$ & $0,33 \pm 1,65$ & $-77 \%$ & .011 \\
Costi ospedalieri & $\$ 4.463 \pm 20.656$ & $\$ 1.912 \pm 7.595$ & $-57 \%$ & .016 \\
Per ricovero & & & & .298 \\
Giorni in ospedale & $10,81 \pm 18,01$ & $7,39 \pm 6,55$ & $-32 \%$ & .049 \\
Giorni in ICU/CCU & $5,61 \pm 18,97$ & $1,49 \pm 2,58$ & $-73 \%$ & .097 \\
Costi ospedalieri & $\$ 16.426 \pm 35.377$ & $\$ 9.318 \pm 11.304$ & $-43 \%$ & \\
\hline
\end{tabular}

\section{Beta-bloccanti}

In particolare, l'uso dei beta-bloccanti è di molto inferiore al desiderabile anche se per questi farmaci esistono documentazioni inoppugnabili della loro efficacia in tutte le classi di scompenso e valutazioni di tipo farmacoeconomico che ne dimostrano il favorevolissimo rapporto costo-beneficio. Una valutazione farmacoeconomica effettuata sull'impiego di carvedilolo [15] ha mostrato come nei soggetti trattati con il beta-bloccante, le giornate di degenza ospedaliera si riducono del $49 \%$, con il $77 \%$ in meno di ricorso alla terapia intensiva e una riduzione del $27 \%$ dei costi ospedalieri complessivi (Tabella IV)

Anche nello studio CIBIS II, l'uso di un altro beta-bloccante, il bisoprololo, ha portato ad una riduzione del $37 \%$ dei ricoveri e a un risparmio economico significativo [16] (Tabella V).

Quanto alle eventuali differenze tra betabloccanti rispetto alla capacità di prevenire le recidive e quindi ridurre i ricoveri (e di conseguenza i costi), è stato dimostrato che le probabilità di riospedalizzazione sono minori nei soggetti trattati con carvedilolo rispetto quelli che ricevono metoprololo [17] (Figura 3) In questo studio, anche se i costi di acquisto del carvedilolo erano superiori a quelli del metoprololo (1.667 dollari vs. 1332 per paziente) i costi totali sono risultati assai inferiori per il carvedilolo (\$ 8.100) rispetto al metoprololo $(\$ 14.475 ; P=.025)$.

I beta-bloccanti sono in grado di influenzare positivamente non solo la qualità di vita dei pazienti scompensati (attraverso il miglioramento della classe funzionale e la riduzione del numero delle ospedalizzazioni), ma anche la sopravvivenza. Già nel 1996 l'US Carvedilol Study mostrava per la prima volta in maniera significativa che un beta-bloccante (carvedilolo) aumenta la sopravvivenza di pazienti con scompenso cardiaco stabile in pazienti in classe II-III [18]. In seguito, gli studi
MERIT HF (metoprololo slow-release) e CIBIS II (bucindololo), confermano che nei pazienti in classe II-III. I beta-bloccanti riducono la mortalità totale rallentando la progressione dello scompenso e prevenendo al morte improvvisa $[19,20]$. Lo studio COPERNICUS [21] conferma la riduzione della mortalità con carvedilolo in soggetti con scompenso grave (classe IV). Questo studio evidenzia che trattando per tre anni 1000 soggetti con scompenso grave si salvano 200 vite. In seguito lo studio CAPRICORN [22] ha dimostrato che il trattamento con carvedilolo in

\begin{tabular}{lcc}
\hline & Placebo & Bisoprololo \\
\hline Francia & FF 35.009 & FF 31.762 \\
Germania & DM 11.563 & DM 10.784 \\
Regno Unito & $£ 4.987$ & $£ 4.722$ \\
\hline
\end{tabular}

\section{Tabella V}

Valutazione dei costi dei pazienti arruolati nello studio CIBIS II in vari paesi europei [16]

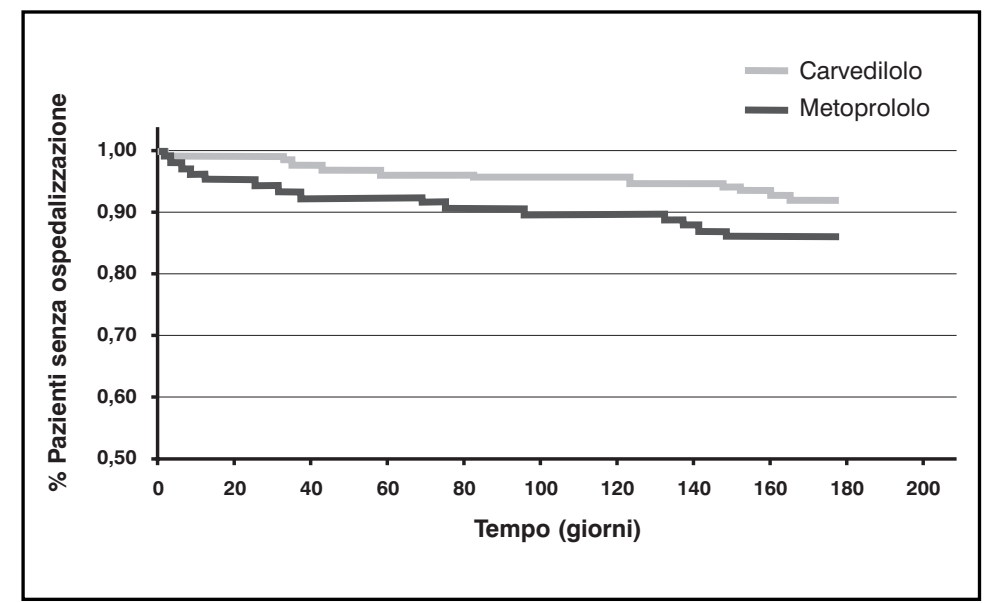

Figura 3

Percentuale di pazienti senza ospedalizzazioni a sei mesi nei trattati con carvedilolo o metoprololo

$\mathrm{P}=.105$

\section{Tabella IV}

Effetto del carvedilolo sui ricoveri in ospedale e sui relativi costi [15]

/CCU = Intensive Care Unit/Critical Care Unit

Le colonne relative a placebo e carvedilolo riportano i dati più o meno la deviazione standard 
soggetti con infarto recente e ridotta frazione d'eiezione $(<40 \%)$ già in terapia con ACEinibitore e con tutti i farmaci ritenuti utili, riduce il rischio di mortalità totale in maniera significativa $(-23 \%)$. Più recentemente lo studio COMET ha per la prima volta confrontato due beta-bloccanti (carvedilolo vs. metoprololo) in soggetti con scompenso cardiaco. Lo studio ha dimostrato che il carvedilolo è superiore al metoprololo nel ridurre la mortalità totale $(-17 \%)$ e che ogni 60 pazienti trattati con carvedilolo si risparmia una vita rispetto a 60 trattati con metoprololo [23]. Al momento gli studi che hanno evidenziato l'efficacia dei beta-bloccanti nello scompenso sono relativi a tre farmaci: metoprololo slow-release, bucindololo e carvedilolo (quest'ultimo è il farmaco con il maggior numero di studi). Essendo i beta-bloccanti una classe di molecole con differenze anche significative, è opportuno che l'impiego clinico nello scompenso venga limitato ai farmaci per cui esistono studi controllati e non agli altri. È interessante notare l'impatto dei vari dosaggi dei singoli beta-bloccanti: nel caso del metoprololo e del bisoprololo l'obiettivo è stato raggiunto rispettivamente con 200 milligrammi/die del primo e 10 milligrammi/die del secondo, mentre il carvedilolo è risultato efficace già alla dose di 6,25 milligrammi due volte al giorno (Studio MOCHA) [24]. I beta-bloccanti sono quindi indicati in tutti i casi di scompenso cardiaco lieve, medio e severo in condizioni di stabilità clinica, dovuto a cardiomiopatia ischemica o non ischemica, con ridotta frazione di eiezione ed in classe NYHA II-IV, in terapia con ACEinibitori e diuretici, a meno di controindicazioni.

I beta-bloccanti sono anche indicati per la terapia a lungo termine in caso di disfunzione ventricolare sinistra asintomatica dopo infarto miocardico. Uno studio recente ha calcolato che l'impiego di beta bloccanti aumenta la sopravvivenza media di 0,3 anni per paziente e riduce i costi sociali di circa 4.000 dollari per paziente in 5 anni [25].

\section{ACE-INIBITORI}

Mentre per i beta-bloccanti non è possibile parlare genericamente di benefici di clas$\mathrm{se}$, in quanto esistono differenze farmacologiche e cliniche anche notevoli tra le varie molecole, diverso è il discorso per quanto riguarda gli ACE-I, che, a dosi equivalenti, producono tutti gli stessi benefici. Tutte le maggiori linee guida raccomandano l'utilizzo di questi farmaci, senza particolare preferenza per una molecola in particolare, nei pazienti con scompenso cardiaco sintomatico, sulla base dell'inoppugnabile evidenza della loro efficacia in termini di riduzione della mortalità e del rischio di ricovero. Numerosi lavori hanno valutato se un approccio basato sulla somministrazione di ACE-I a tutti i pazienti con insufficienza cardiaca, oltre a essere efficace, fosse anche costo-efficace e sostenibile dal punto di vista finanziario. Glick e coll. [26], ad esempio, hanno trasferito i dati clinici da loro ottenuti nello studio SOLVD in un modello per l'analisi decisionale, alimentato con costi statunitensi, per valutare gli outcome clinici ed economici della somministrazione di ACEI (nella fattispecie l'enalapril) a breve e a lungo termine. Il modello matematico ha stimato che nei 48 mesi di durata dello studio il trattamento con enalapril risulta in un guadagno medio di 0,16 anni di vita per paziente, con un concomitante risparmio di circa 700 US\$, configurando la situazione di dominanza farmacoeconomica (miglior efficacia ad un costo minore). Proiettando i benefici e i costi sull'intera durata residua della vita dei pazienti arruolati, il modello stima un vantaggio medio di 0,40 anni di sopravvivenza, ad un costo di 80 US\$ per anno di vita salvato o di 115 US\$ per QALY (anno di vita ponderato per la qualità) con l'uso dell' ACE-I, situando la terapia dello scompenso con queste molecole ai primi posti di un'ipotetica classifica degli interventi più costo-efficaci in cardiologia.

L'influenza della terapia con ACE-I sulla formazione dei costi sanitari sostenuti per il trattamento dei pazienti con insufficienza cardiaca è stata anche analizzata in un setting non sperimentale, attraverso l'esame retrospettivo delle richieste di rimborso per spese farmaceutiche e mediche di 1.573 pazienti inclusi in un programma di assistenza sanitaria statunitense [27]. Dopo correzione per i fattori potenzialmente confondenti, i pazienti esposti alla terapia con ACE-I hanno mostrato un rischio significativamente minore di essere ricoverati in ospedale ( $R R 0,65 ; p<0,0001)$ e hanno assorbito meno risorse complessive, con un costo medio totale significativamente inferiore $(-2.397$ US\$, $\mathrm{p}<0.001)$, a dispetto del superiore costo farmaceutico sostenuto.

Anche per gli ACE-I valgono quindi le considerazioni già fatte a proposito dei betabloccanti: il problema principale da tenere sotto osservazione non è tanto l'eccessiva spesa farmaceutica, ma semmai la scarsa utilizzazione dei farmaci, intesa sia come mancata prescrizione, sia come utilizzo di dosi troppo basse. Quest'ultimo aspetto è stato indagato dallo studio ATLAS [28], un trial multicentrico randomizzato e controllato condotto su oltre 3.000 pazienti con scompenso di classe NYHA II-IV e frazione di eiezione ventricolare $\leq 30 \%$ che ha messo a confronto un regime a base di alte dosi (32,5-35 $\mathrm{mg} / \mathrm{die})$ di lisinopril, vicino a quello utilizzato nei trial e raccomandato dalle linee guida, con una 
posologia inferiore (2,5-5 mg/die), più prossima a quella prescritta nella pratica clinica. I pazienti assegnati al dosaggio pieno hanno avuto meno ospedalizzazioni e meno giorni di degenza, e tali riduzioni sono risultate statisticamente significative in particolare per i ricoveri attribuiti direttamente allo scompenso. Nei pazienti trattati ad alte dosi di ACE-I, la riduzione dei costi ospedalieri ha compensato l'incremento di spesa farmaceutica, con il risultato netto di un miglioramento degli outcome clinici senza aumento della spesa sanitaria sostenuta per la loro gestione.

Come regola generale, la costo-efficacia di un trattamento tende ad aumentare insieme alla gravità della malattia, a causa del minor numero di persone che vanno trattate (NNT - number needed to treat) per riuscire ad evitare un evento clinico (ad esempio, un ricovero per scompenso). Ciò, in una situazione di risorse limitate che impone di effettuare scelte politiche sui pazienti a cui offrire la terapia, comporta la tendenza a privilegiare quelli più gravi, anche se non sempre questa logica coincide con le priorità cliniche. Lo studio SOLVD, infatti, ha dimostrato che il trattamento con ACE-I è in grado di ridurre la morbilità e la mortalità anche nei pazienti con ridotta frazione di eiezione ancora asintomatici, che normalmente non vengono trattati con questi farmaci almeno fino allo sviluppo di insufficienza cardiaca manifesta. Sulla base di questi dati, l'agenzia governativa statunitense per la ricerca e la qualità dell'assistenza sanitaria (AHRQ - Agency for Healthcare Research and Quality) ha commissionato uno studio per indagare, oltre ad altri aspetti della terapia dello scompenso cardiaco, la convenienza ad adottare una strategia aggressiva nei pazienti con disfunzione ventricolare, basata sulla somministrazione di ACE-I a tutti i pazienti con $\mathrm{FE}<35 \%$, in presenza o in assenza di sintomatologia. A tal fine, gli autori hanno modellizzato gli esiti clinici ed economici a lungo termine di un'ipotetica coorte di pazienti 55enni trattati con due strategie alternative, di cui la prima prevedeva la terapia a base di ACE-I dal momento della diagnosi di ridotta funzionalità ventricolare, mentre l'altra posticipava il ricorso a queste molecole fino alla comparsa della sintomatologia. I risultati della simulazione del modello indicano che, nel corso dell'intera durata della loro vita residua (media di 8,1 anni, con un tasso di mortalità e morbilità a 5 anni del $57 \%$ nei non trattati), i pazienti trattati precocemente hanno un guadagno medio di 8 mesi, in termini sia di sopravvivenza sia di sopravvivenza ponderata per la qualità di vita, ad un costo incrementale di 3.718 US\$. Trasformati in rapporti di costo/efficacia marginale, questi risultati determinano valori di $\$ 5.802$ US $\$ /$ anno di vita guadagnato e di \$5.644/QALY guada- gnato, dunque ampiamente entro i limiti considerati accettabili per le società avanzate, che convenzionalmente fissano in 20.000 US\$/ QALY la soglia degli interventi sanitari considerati molto convenienti.

Poiché l'individuazione sistematica di pazienti asintomatici richiede un metodo di screening, gli autori della ricerca hanno esteso l'analisi a un ulteriore scenario, in cui vengono confrontate le varie strategie di screening alternative. I risultati indicano che in una popolazione di 55enni asintomatici (prevalenza di frazione di eiezione ridotta stimata al 2,7\%) i migliori risultati clinici si otterrebbero con l'utilizzo dell' ecocardiografia per lo screening universale. La gestione di questa popolazione con una strategia basata su uno screening iniziale con il BNP, seguito dall' ecocardiografia e dalla terapia con ACEI nei casi positivi, migliorerebbe significativamente i risultati clinici, ad un costo di 18.000 euro/QALY guadagnato rispetto all'assenza di screening [29].

\section{Diuretici}

La terapia diuretica ha costituito per lungo tempo un pilastro del trattamento dello scompenso cardiaco. Insieme alla terapia digitalica, è stata ampiamente usata per risolvere le crisi di scompenso cardiaco acuto e nella terapia di mantenimento [30]. Le due principali classi di diuretici disponibili sono quella ad azione sul tubulo distale e quella ad azione sull'ansa ascendente di Henle. Alla prima appartengono i diuretici tiazidici, clortalidone e indapamide. Questi inducono un modesto incremento della natriuresi, compreso fra 5 e $10 \%$ del carico filtrato, e la loro efficacia viene nettamente ridotta in presenza di insufficienza renale, con filtrato glomerulare inferiore a $30 \mathrm{ml} /$ minuto. Viceversa i diuretici attivi sull'ansa di Henle (furosemide e simili) provocano una natriuresi che può raggiungere il $25 \%$ del filtrato e sono in grado di mantenere la loro efficacia anche per valori di filtrato fino a $5 \mathrm{ml} / \mathrm{min}$. L'associazione fra due diuretici a diverso sito di azione incrementa ulteriormente la diuresi. Negli ultimi anni l'arsenale terapeutico a disposizione del medico per la cura del paziente scompensato si è notevolmente arricchito, con l'avvento di ACEinibitori, beta-bloccanti, e inibitori dell'angiotensina II, che nel complesso hanno contribuito a ridurre la mortalità, le riospedalizzazioni, le recidive e la morbilità correlata a questa patologia. Benché l'uso dei diuretici sia tuttora fondamentale nella risoluzione dello scompenso cardiaco acuto, il loro ruolo è stato ridimensionato nella fase di mantenimento, parallelamente ad una maggior conoscenza del quadro fisiopatologico alla base dello scompenso e del ruolo dell' attivazione del sistema neurormonale nel paziente 
con scompenso cronico. Questo sistema, infatti, reagisce alla terapia diuretica con l'aumento del tono adrenergico e con l'attivazione del sistema renina-angiotensina. Questo è il motivo per cui i diuretici non permettono di mantenere a lungo una stabilità emodinamica e di aumentare la sopravvivenza nei pazienti scompensati [31]. La terapia diuretica, tuttavia, è molto utile per il controllo della sintomatologia, grazie alla riduzione della pressione di riempimento del ventricolo destro e della pressione arteriosa polmonare, ed in misura minore di quelle del ventricolo sinistro e arteriosa sistemica, cui consegue un miglioramento dei sintomi di congestione, come la dispnea. D'altro canto, una diuresi eccessiva può ridurre la pressione venosa e la pressione di riempimento ventricolare, con riduzione della gittata cardiaca, ridotta perfusione tessutale, ipotensione arteriosa e segni di disidratazione. In particolare nell' anziano questo quadro clinico può essere responsabile di sincope, emoconcentrazione e insufficienza renale. Per questo motivo uno schema fisso di dosaggio è chiaramente insoddisfacente. Il dosaggio dei diuretici nella fase di mantenimento deve essere aggiustato in accordo con le necessità cliniche e l'idratazione del singolo paziente.

Anche i farmaci antialdosteronici, spironolattone o il suo metabolita canreonato, si sono dimostrati utili, a basse dosi, nell'aumentare la sopravvivenza in pazienti con scompenso cardiaco congestizio, e nel ridurre recidive di scompenso e riospedalizzazione [32].

Le nuove opzioni farmacologiche hanno pertanto ridefinito il ruolo della terapia diuretica, limitandola al controllo e alla riduzione della ipervolemia nello scompenso congestizio cronico, in associazione con farmaci che riducano l'attivazione neurormonale. La relativa diminuzione dell'interesse rivolto al ruolo dei diuretici nella terapia dello scompenso cronico, e in particolare l'assenza di evidenze scientifiche robuste che ne dimostrino $\mathrm{i}$ benefici in termini di mortalità $\mathrm{o}$ riduzione delle ospedalizzazioni, sono molto probabilmente la causa dell'assenza di studi farmacoeconomici di rilevo che ne analizzino l'impatto complessivo sul costo di malattia.

Nel tentativo di razionalizzare il mercato farmaceutico e contenerne la spesa a carico del SSN, negli ultimi anni sono state introdotte alcune innovazioni normative che hanno creato le condizioni necessarie per favorire lo sviluppo del mercato dei generici anche in Italia. Ogni prodotto farmaceutico, allo scadere della protezione brevettuale, può infatti essere riprodotto e commercializzato da azien- de farmaceutiche concorrenti. I farmaci "copia" possono essere registrati e commercializzati con un nome di fantasia ("generici branded") o con la denominazione comune internazionale (DCI) seguita dal nome del produttore (generici propriamente detti).

Gli obiettivi principali della politica del generico sono di favorire un mercato farmaceutico maggiormente aperto alla concorrenza, di innescare una dinamica di riduzione dei prezzi dei medicinali e di consentire significativi risparmi sulla spesa farmaceutica pubblica permettendo, in ultima analisi, di liberare risorse da destinare ai farmaci innovativi più costosi. In Italia questi obiettivi vengono perseguiti mediante l'adozione di tre norme: a) il prezzo del generico deve essere almeno il $20 \%$ inferiore a quello del prodotto “originatore". L'"originatore" può, a sua volta, diminuire il prezzo anche sotto il livello dei generici, innescando in tal modo una dinamica di prezzi al ribasso grazie alla possibilità di entrare in un mercato concorrenziale; b) il prezzo del generico, o il prezzo più basso tra quelli dei prodotti generici equivalenti, viene assunto come prezzo di riferimento rimborsabile dal SSN per quel farmaco; c) il farmacista è tenuto a dispensare un "generico" in sostituzione di un equivalente prodotto di marchio, salvo il caso in cui il medico specifichi il contrario o il paziente sia disposto a pagare l'eventuale differenza rispetto alla quota rimborsata dal SSN [33].

In Italia il mercato dei generici è iniziato da meno di 5 anni ed ha rivelato una dinamica di riduzione dei prezzi su base concorrenziale discretamente attiva, comportando per alcuni prodotti un abbattimento del prezzo iniziale di oltre il $50 \%$.

In questi anni il mercato dei generici ha raggiunto quote sensibili, benché non straordinarie, e si tratta di un mercato in espansione, sia come volume complessivo di confezioni vendute sia come introduzione di nuovi prodotti. Il numero di prodotti generici disponibile è infatti relativamente elevato, particolarmente nelle patologie ad elevata prevalenza, come nel caso dello scompenso cardiaco (Tabella VI), ma anche dell'ansia, della depressione, del diabete, ecc.

È dunque oggi possibile gestire intere popolazioni di pazienti, in accordo con le raccomandazioni delle linee guida, utilizzando i prodotti generici, con un contenimento dei costi e, soprattutto, un'amplificazione dei benefici, clinici ed economici, descritti nei paragrafi precedenti.

Per quanto riguarda i farmaci utilizzati nel trattamento dello scompenso cardiaco, l'introduzione dei generici ha comportato una significativa riduzione dei prezzi, prossima al 50\%. Nella Tabella VII abbiamo valorizzato in termini monetari uno schema posologico "tipico" per un paziente scompensato trattato in accor- 
do alle linee guida (carvedilolo $25 \mathrm{mg}$ due volte al dì + enalapril $20 \mathrm{mg} / \mathrm{die}+$ potassio canreonato $100 \mathrm{mg} / \mathrm{die}$ ), in base ai prezzi vigenti prima dell'introduzione del rispettivo generico e a quelli attuali.

Come si può osservare, il costo farmaceutico di un tale schema posologico si è ridotto del $45 \%$ dall'introduzione del generico. Abbiamo già discusso come la spesa per farmaci rappresenti una parte minore del costo complessivo dello scompenso cardiaco e che sarebbe necessario affrontare il tema della riduzione dei costi di malattia con approcci rivolti ad altre componenti di spesa.

\begin{tabular}{|c|c|c|c|}
\hline Codice ATC & Farmaco & Confezione & Prezzo di riferimento (euro) \\
\hline \multicolumn{4}{|c|}{ Beta-bloccanti non associati } \\
\hline \multirow[t]{2}{*}{ C07AA07 } & Sotalolo & 40 unità 80 mg & 4,70 \\
\hline & & 50 unità 80 mg & 5,68 \\
\hline \multirow[t]{6}{*}{ C07AB02 } & Metoprololo & 100 unità 100 mg & 16,62 \\
\hline & & 28 unità 200 mg & 8,69 \\
\hline & & 30 unità 100 mg & 4,03 \\
\hline & & 30 unità 200 mg & 7,01 \\
\hline & & 50 unità 100 mg & 8,02 \\
\hline & & 50 unità 200 mg & 13,02 \\
\hline \multirow[t]{4}{*}{ С07AB03 } & Atenololo & 14 unità 100 mg & 3,10 \\
\hline & & 42 unità 100 mg & 6,75 \\
\hline & & 50 unità 100 mg & 8,35 \\
\hline & & 50 unità 50 mg & 6,01 \\
\hline \multirow[t]{2}{*}{ C07AG02 } & Carvedilolo & 28 unità $6,25 \mathrm{mg}$ & 5,00 \\
\hline & & 30 unità 25 mg & 11,50 \\
\hline \multicolumn{4}{|c|}{ Beta-bloccanti associati a diuretici } \\
\hline \multirow[t]{4}{*}{ С07СВ03 } & Atenololo+Clortalidone & 28 unità $(100+25)$ mg & 8,00 \\
\hline & & 28 unità $(50+12,5)$ mg & 4,10 \\
\hline & & 30 unità $(100$ + 25) mg & 8,52 \\
\hline & & 30 unità $(50+12,5) \mathrm{mg}$ & 4,91 \\
\hline \multicolumn{4}{|l|}{ Ace-inibitori } \\
\hline \multirow[t]{2}{*}{ C09AA01 } & Captopril & 24 unità 50 mg & 7,33 \\
\hline & & 50 unità 25 mg & 8,12 \\
\hline \multirow[t]{2}{*}{ C09AA02 } & Enalapril & 14 unità 20 mg & 5,50 \\
\hline & & 28 unità 5 mg & 5,00 \\
\hline \multicolumn{4}{|l|}{ Diuretici } \\
\hline \multirow[t]{2}{*}{ C03CA01 } & Furosemide & 20 unità 500 mg & 17,20 \\
\hline & & 30 unità 25 mg & 1,62 \\
\hline C03CA04 & Torasemide & 14 unità 10 mg & 2,80 \\
\hline \multirow[t]{2}{*}{ C03BA11 } & Indapamide & 30 unità 2,5 mg & 4,49 \\
\hline & & 50 unità 2,5 mg & 7,00 \\
\hline C03DA02 & Potassio canrenoato & 20 unità 100 mg & 4,80 \\
\hline C03AA03 & Idroclorotiazide & 20 unità 25 mg & 1,29 \\
\hline
\end{tabular}

Tabella VI

Confezioni e prezzi dei beta-bloccanti, ACE-inibitori e diuretici disponibili come generici in Italia [33] 


\begin{tabular}{lcccc}
\hline & Carvedilolo & Enalapril & Potassio Canreonato & TOTALE \\
\hline Confezione & 30 unità $25 \mathrm{mg}$ & 14 unità $20 \mathrm{mg}$ & 20 unità $100 \mathrm{mg}$ \\
Prezzo pre-generico (euro) & 19,8 & 11,3 & 8,3 \\
Prezzo dal generico (euro) & 11,5 & 5,5 & 4,8 & $760 \mathrm{mg}$ uid \\
Posologia valorizzata & $25 \mathrm{mg}$ bid & $20 \mathrm{mg}$ uid & 12,5 & 42 \\
Costo mensile pre-generico (euro) & 39,5 & 24,2 & 7,2 & $-45 \%$ \\
Costo mensile con generico (euro) & 23 & 11,8 & & $82 \%$ \\
\hline $\begin{array}{l}\text { Delta costi } \\
\text { Pazienti aggiuntivi trattabili senza } \\
\text { eccesso di spesa }\end{array}$ & & & & \\
\hline
\end{tabular}

\section{Tabella VII}

Confronto dei costi/terapia dello scompenso cardiaco prima e dopo l'introduzione dei generici (per spiegazioni più dettagliate si rimanda al testo)

Potrebbe dunque sembrare paradossale insistere sulla riduzione della spesa farmaceutica, vista la capacità dei farmaci di ridurre i costi associati. Riteniamo infatti che la chiave di lettura corretta sia un'altra e che il dato più interessante sia riportato nell'ultima colonna della Tabella VII: a parità di spesa farmaceutica, come noto regolata da norme su tetti di spesa e sforamenti che ne rendono difficile l'incremento anche a fronte della dimostrazione di un beneficio economico complessivo, è oggi possibile trattare più pazienti con scompenso cardiaco $(+82 \%$, o 182 ogni 100 pazienti trattati ai prezzi pre-generico), estendendo le positive ricadute cliniche ed economiche di una terapia efficace a un numero maggiore di pazienti.

\section{CONCLUSIONI}

Lo scompenso cardiaco è una patologia grave che induce un elevato consumo di risorse sanitarie. L'elevata prevalenza comporta un pesante onere economico per tutte le società industrializzate, che si prevede ancora maggiore nei prossimi anni, sia per l'aumento dell'incidenza, sia per l'aumentata sopravvivenza dei pazienti. Il principale costdriver dello scompenso cardiaco è rappre- sentato dai frequenti ricoveri ospedalieri per recidiva. La prima causa di riacutizzazione dell'insufficienza cardiaca è un trattamento inadeguato, inteso sia come compliance insufficiente allo schema terapeutico prescritto, sia come prescrizione di terapie sub-ottimali, a dispetto delle ampie evidenze di efficacia delle terapie oggi previste dalle linee guida, in gran parte basate su ACE-inibitori, diuretici e beta-bloccanti. Beta-bloccanti e ACE-inibitori, inoltre, sono stati studiati anche dal punto di vista farmacoeconomico ed è dimostrato che il loro utilizzo è in grado non solo di aumentare la sopravvivenza e migliorare la sintomatologia, ma anche di ridurre in misura sensibile le recidive e quindi i ricoveri, con l'effetto di ottenere un risparmio netto sul costo globale di patologia. Occorre poi ricordare che i betabloccanti e gli ACE-inibitori considerati il gold standard nella terapia dello scompenso sono oggi privi di copertura brevettuale e che, essendosi ridotto significativamente il prezzo d'acquisto in seguito all'introduzione dei prodotti generici, essi possono essere offerti a un maggior numero di pazienti senza incrementare la spesa farmaceutica, rendendo ancor più rilevanti i vantaggi economici del loro impiego per il SSN e la società nel suo complesso. 


\section{BIBLIOGRAFIA}

1. Ho KK, Pinsky JL, Kannel WB, Levy D. The epidemiology of heart failure: the Framingham Study. J Am Coll Cardiol 1993; 22 (Suppl A): 6A-13A.

2. Cowie MR, Mosterd A, Wood DA, et al. The epidemiology of heart failure. Eur Heart J 1997; 18: 208-25.

3. Remme WJ, Swedberg K, for the Task Force for the Diagnosis and Treatment of Chronic Heart Failure, European Society of Cardiology. Guidelines for the diagnosis and treatment of chronic heart failure. Eur Heart J 2001; 22: 1527-60.

4. Ministero della Sanità. Sistema informativo sanitario. Scheda di Dimissione Ospedaliera 1999.

5. Krumholz HM, Parent EM, Tu N, et al. Readmission after hospitalization for congestive heart failure among Medicare beneficiaries. Arch Intern Med 1997; 157: 99-104.

6. Gooding J, Jette AM. Hospital readmissions among the elderly. J Am Geriatr Soc 1985; 33: 596-601.

7. McMurray J, Hart W, Rhodes G. An evaluation of the cost of heart failure to the National Health Service in the UK. Br Med Econ 1993; 6: 99-110.

8. Politi C, Deales A, Cicchitelli F. Lo scompenso cardiaco nelle Marche: assorbimento delle risorse. 2004, Atti del Convegno AIES, Milano 4-5 Novembre 2004

9 CeVEAS: Centro per la Valutazione della Efficacia della Assistenza SanitariaA.S.S. N.6 FRIULI OCCIDENTALE Pordenone A.O. S. MARIA DEGLI ANGELI Pordenone A.P.S.S Trento: Gestione della Insufficienza Cardiaca, Agosto 2001

10. Task Force for the Diagnosis and Treatment of Chronic Heart Failure, European Society of Cardiology. Guidelines for the diagnosis and treatment of chronic heart failure. Eur Heart J 2001; 22: 1527-60.

11. Hunt SA, Baker DW, Chin MH, et al. American College of Cardiology/American Heart Association Task Force on Practice Guidelines. ACC/AHA guidelines for the evaluation and management of chronic heart failure in the adult. J Am Coll Cardiol 2001; 38: 2101-13.

12. Database dell'Italian Network on Congestive Heart Failure (IN-CHF): risultati e prospettive. Atti del Convegno di Napoli-2000

13. Komajda M, Lapuerta P, Hermans $\mathrm{N}$ et al. Adherence to guidelines is a predictor of outcome in chronic heart failure:the MAHLER survey. Eur Heart J. 2005 Apr 12;

14. Valle R, Carbonieri E, Tenderini P et al. Una proposta di protocollo per la gestione ambulatoriale dei pazienti dimessi con diagnosi di scompenso cardiaco: il progetto collaborativo Venetia. HF Ital Heart J. 2004, 282-291 Suppl Vol 5

15. Fowler MB, Vera-Llonch M, Oster G, et al. Influence of carvedilol on hospitalizations in heart failure: incidence, resource utilization and costs. J Am Coll Cardiol. 2001;37:1692-1699.

16. CIBIS-II Investigators and Committees. Reduced costs with bisoprolol treatment for heart failure: an economic analysis of the second Cardiac Insufficiency Bisoprolol Study (CIBIS-II). Eur Heart J. 2001;22:1021-1031.

17. Luzier AB, Antell LA, Chang LL, et al. Reimbursement claims analysis of outcomes with carvedilol and metoprolol. Ann Pharmacother. 2002;36:386-391.

18. Packer M, Bristow MR, Cohn JN et al. The effect of carvedilol on morbidity and mortality in patients with chronic heart failure. N Engl J Med 1996; 334: 1349-55

19. The Merit-HF Study Group. Effect of metoprolol CR/XL in chronic heart failure: metoprolol CR/XL randomised intervention trial in congestive heart failure (MERIT-HF). Lancet 1999; 353: 2001-7

20. Cibis II Investigators and Committees. The Cardiac Insufficiency Bisoprolol Study II (CIBIS II): a randomised trial. Lancet 1999; 353: 9-13

21. Packer M, Fowler MD, Roecker EB, et al. Effectof carvedilol on the morbidity of patients with severe chronic heart failure: results of the carvedilol prospective randomized cumulative survival study (COPERNICUS). Circulation.2002;106:2194-2199.

22. The CAPRICORN Investigators. Carvedilol post infarction survival control in left ventricular dysfunction (CAPRICORN). Lancet. 2001; 357:1385-1390.

23. Poole-Wilson PA, Swedberg K, Cleland JGF, etal. Comparison of carvedilol and metoprolol on clinical outcomes in patients with chronic heart failure in the carvedilol or metoprolol (COMET) randomized controlled trial. Lancet. 2003;362:7-13. 
24. Bristow MR, Gilbert EM, Abraham WT et al. for the MOCHA Investigators. Carvedilol produces dose-related improvements in left ventricular function and survival in subjects with chronic heart failure. Circulation 1996; 94: 2807-16

25. Cowper PA, De Long ER, Whellan DJ et al.Economic effects of beta-blocker therapy in patients with heart failure. Am J Med. 2004 Jan 15;116(2):104-11.

26. Glick H, Cook J, Kinosian B, et al. Costs and effects of enalapril therapy in patients with symptomatic heart failure: an economic analysis of the Studies of Left Ventricular Dysfunction (SOLVD). Treatment Trial. J Card Fail. 1995 Dec;1(5):371-80.

27. Abarca J, Malone DC, Armstrong EP, Zachry WM $3^{\text {rd }}$ Angiotensin-converting enzyme inhibitor therapy in patients with heart failure enrolled in a managed care organization: effect on costs and probability of hospitalization. Pharmacotherapy. 2004 Mar;24(3):351-7.

28. Schwartz JS, Wang YR, Cleland JG, et al. High-versus low-dose angiotensin converting enzyme inhibitor therapy in the treatment of heart failure: an economic analysis of the Assessment of Treatment with Lisinopril and Survival (ATLAS) trial. Am J Manag Care. 2003 Jun;9(6):417-24.

29. Shekelle P, Rich M, Morton S, et al. Pharmacologic Management of Heart Failure and Left Ventricular Systolic Dysfunction: Effect in Female, Black, and Diabetic Patients, and Cost-Effectiveness. Evidence Report/Technology Assessment No. 82 (Prepared by the Southern California-RAND Evidence-based Practice Center under Contract No 290-97-0001). AHRQ Publication No. 03-E045. Rockville, MD: Agency for Healthcare Research and Quality. July 2003.

30. The Digitalis Investigation Group. The effect of digoxin on mortality and morbidity in patients with heart failure. NEngl J Med 1997; 336: 525-533.

31. Cecchi F. I diuretici dell' ansa nella terapia di mantenimento del paziente con scompenso cardiaco. Farmeconomia e percorsi terapeutici 2004; 5 (3): 133-140

32 Pitt B, Sannad F, Remme WJ et al. The effect of spironolactone on morbidity and mortality in patients with severe heart failure. Randomized aldactone evaluation study investigators. N Engl J Med 1999; 341: 709-17.

33. Eandi M, Della Pepa C. I farmaci "generici” in Italia: opportunità di ricerca e sviluppo di prodotti di qualità a prezzi competitivi. Farmeconomia e percorsi terapeutici 2003; 4 (2): 65-76

34. http://www.ministerosalute.it/medicinali/generici/pdf_files/Generici_Prontuario_NewPrezziRif_PA.pdf, aggiornamento al $11 / 05 / 05$ 